Navier-Stokes Neutral and

Plasma Fluid Modelling in 3D

J. Riemann, M. Borchardt, R. Schneider, A.

Mutzke, T.D. Rognlien, M. Umansky

May 17, 2004

Contributions to Plasma Physics 
This document was prepared as an account of work sponsored by an agency of the United States Government. Neither the United States Government nor the University of California nor any of their employees, makes any warranty, express or implied, or assumes any legal liability or responsibility for the accuracy, completeness, or usefulness of any information, apparatus, product, or process disclosed, or represents that its use would not infringe privately owned rights. Reference herein to any specific commercial product, process, or service by trade name, trademark, manufacturer, or otherwise, does not necessarily constitute or imply its endorsement, recommendation, or favoring by the United States Government or the University of California. The views and opinions of authors expressed herein do not necessarily state or reflect those of the United States Government or the University of California, and shall not be used for advertising or product endorsement purposes. 


\title{
Navier-Stokes Neutral and Plasma Fluid Modelling in 3D
}

\author{
J. Riemann*1 ${ }^{*}$, M. Borchardt ${ }^{1}$, R. Schneider ${ }^{1}$, A. Mutzke ${ }^{1}$, T. D. Rognlien ${ }^{2}$, and M. Umansky ${ }^{2}$ \\ ${ }^{1}$ Max-Planck-Institut für Plasmaphysik, EURATOM Assoc., Wendelsteinstr.1, 17491 Greifswald, Germany \\ ${ }^{2}$ Lawrence Livermore Nat. Lab., CA 94551 Livermore, U.S.A.
}

Key words Plasma edge, finite volume, neutral fluid, Navier-Stokes.

PACS 02.60.Cb, 02.60.Lj, 52.25.Ya

The 3D finite volume transport code BoRiS is applied to a system of coupled plasma and neutral fluid equations in a slab. Demonstrating easy implementation of new equations, a new parallel BoRiS version is tested on three different models for the neutral fluid - diffusive, parallel Navier-Stokes and full Navier-Stokes - and the results are compared to each other. Typical effects like density enhancement by ionization of recycled neutrals in front of a target plate can be seen and differences are linked to the neutral models in use.

\section{Introduction}

Coupled modelling of plasma and neutral fluids is a standard technique for the description of edge plasma phenomena in fusion devices. An exact treatment requires the solution of three Navier-Stokes equations for the neutral momentum. However, in most cases only a simplified treatment of the neutral particle momentum is used. Such a simplified ansatz considers a 1D Navier-Stokes equation for the neutral momentum parallel to the magnetic field while the perpendicular momentum is assumed to be driven by the neutral particle pressure and the corresponding velocities are determined in the framework of a diffusive model.

In this work, the 3D scrape-off layer (SOL) transport code BoRiS is tested on three different neutral models (diffusive, parallel Navier-Stokes and full Navier-Stokes). By doing so, we want to demonstrate easy implementation of new equations and the capability of solving complex systems.

The tests are done with a standard SOL physics model and equations for the plasma (electrons - $e$, ions $-i$ ) and the neutrals $(0)[1,2]$. Both ions and neutrals represent the heavy species $(a=i, 0)$ having the same (hydrogen) mass $m_{0}=m_{i}$ and the same temperature $T_{0}=T_{i}$.

With parallel $(\|)$ and perpendicular $(\perp)$ velocities according to $\vec{V}=\vec{V}+\overrightarrow{V_{\perp}}=\vec{u}+\vec{v}$, we have the following set of equations:

$$
\begin{aligned}
& \frac{\partial}{\partial t}\left(n_{a}\right)+\vec{\nabla} \cdot\left(n_{a} \vec{V}_{a}\right)=S_{n}^{a} \\
& \frac{\partial}{\partial t}\left(m_{a} n_{a} u_{a}\right)+\vec{\nabla} \cdot\left(m_{a} n_{a} u_{a} \vec{V}_{a}-\eta_{a} \vec{\nabla} u_{a}\right)=-\nabla_{\|} p_{a}+S_{m \|}^{a} \\
& \frac{\partial}{\partial t}\left(m_{0} n_{0} v_{0}\right)+\vec{\nabla} \cdot\left(m_{0} n_{0} v_{0} \vec{V}_{0}-\eta_{0} \vec{\nabla} v_{0}\right)=-\nabla_{\perp} p_{0}+S_{m \perp}^{0} \\
& \frac{\partial}{\partial t}\left(\frac{3}{2} p_{e}\right)+\vec{\nabla} \cdot\left(\frac{5}{2} T_{e} n_{e} \vec{V}_{e}-\kappa_{e} \vec{\nabla} T_{e}\right)=u_{e} \nabla_{\|} p_{e}+Q_{e i}+S_{q}^{e}
\end{aligned}
$$




$$
\frac{\partial}{\partial t}\left(\frac{3}{2} \sum_{a} p_{a}\right)+\vec{\nabla} \cdot\left(\frac{5}{2} T_{i} n_{a} \vec{V}_{a}-\sum_{a} \kappa_{a} \vec{\nabla} T_{i}\right)=u_{a} \nabla_{\|} p_{a}-Q_{e i}+S_{q}^{i}
$$

The above equations are written with respect to the physically relevant directions defined by the left-handed curvilinear system $\vec{B}, \vec{\nabla} s, \vec{\nabla} s \times \vec{B}\left(\|, \perp_{1}, \perp_{2}\right)$, where $\vec{B}$ is the magnetic field which forms magnetic surfaces $\vec{\nabla} s$. The plasma properties $n_{i}=n_{e}, u_{i}, T_{e}$ and $T_{i}$ are described by the equations (1) - plasma continuity, (2) plasma parallel momentum, (4) - electron internal energy and (5) - ion-neutral internal energy respectively. The perpendicular plasma velocities follow from $\vec{V}_{\perp, i}=\vec{v}_{i}=-D_{\perp} \vec{\nabla}_{\perp} n_{i}$, with $D_{\perp}$ being the anomalous diffusivity. For full Navier-Stokes neutrals $(a=0)$ in this slab setup, we have the equations (1) - neutral continuity, (2) and (3) - neutral parallel and perpendicular momentum respectively, again together with (5) - ion-neutral internal energy. The above equations contain the viscosities $\eta_{a}$, thermal conductivities $\kappa_{e, i}$ and sources $S_{n}^{a}, S_{m \|}^{a}, S_{m \perp}^{0}$ and $S_{q}^{e, i}$ which account for ionization and charge exchange. $Q_{e i}$ is the heat exchange between ions and electrons. If we assume the propagation of neutrals only being driven by their thermal pressure, equations (2) and (3) are replaced by the neutral velocity in the diffusive model

$$
\vec{V}_{0}=\vec{u}_{0}+\vec{v}_{0}=-D_{0}\left(\vec{\nabla} n_{0} / n_{0}+\vec{\nabla} T_{i} / T_{i}\right)
$$

The more advanced parallel Navier-Stokes model then solves equation (2) for $u_{0}$ and determines $v_{0}$ from the corresponding part of (6).

\section{Setup and boundary conditions}

Our setup is motivated by an experiment/simulation as described in [3]. We consider a slab with dimensions $L_{x}=0.1 \mathrm{~m}, L_{y}=0.1 \mathrm{~m}$ and $L_{z}=4.0 \mathrm{~m}$. In BoRiS, this slab in real space is internally represented by a corresponding $10 \times 6 \times 25$ grid in generalized (magnetic) coordinates $(s, \theta, \phi)$ which are normalized to unity. For simplicity, the magnetic field is along the $z$ axis and $x, y, z$ are perfectly aligned with $s, \theta, \phi$ respectively.

The boundary conditions are as follows:

The $\phi=0(z=0 \mathrm{~m})$ boundary represents a wall with zero-flux conditions and a slit along $\theta(y)$ around the center at $s=0.5(x=0.05 \mathrm{~m})$. For the slit region both electron and ion temperatures are fixed to $T_{e}=T_{i}=15 \mathrm{eV}$. The plasma parallel momentum flux is set to zero and similar to the experimental situation the plasma density varies between $3 \cdot 10^{18} \mathrm{~m}^{-3}(\theta=0)$ and $2.7 \cdot 10^{18} \mathrm{~m}^{-3}(\theta=1)$ thus introducing a 3D effect. At $\phi=0$ the neutral parallel velocity is set to zero while both the neutral density and the neutral perpendicular velocities have zero-gradient conditions.

The $\phi=1(z=4 \mathrm{~m})$ boundary represents a target plate with appropriate sheath conditions for all quantities. Accordingly, $T_{e}$ and $T_{i}$ are determined by their corresponding heat fluxes $\Gamma_{q}^{e, i}=\delta_{e, i} T_{e, i} \Gamma_{n}$ with $\Gamma_{n}$ being the particle flux and the sheath coefficients $\delta_{e}=5$ and $\delta_{i}=3.5$. For the ion heat flux, the additional contribution $\Gamma_{q}^{i}=-1.5\left(T_{e}+T_{i}\right) \Gamma_{n} R_{E}$ accounts for energy recycled by the neutrals with a recycling coefficient $R_{E}=0.3$. The plasma parallel velocity is set to $u_{i}=c_{s}\left(c_{s}=\left(\left(T_{e}+T_{i}\right) / m_{i}\right)^{1 / 2}\right.$ local ion accoustic speed). The neutral particle flux at the plate is $\Gamma_{0}=-R_{0} \Gamma_{n}$ and the neutral parallel velocity is set to $u_{0}=-R_{M} u_{i}$ with particle and momentum recycling coefficients $R_{0}=1.0$ and $R_{M}=0.3$ respectively. Again, we choose zero-gradients for the neutral perpendicular velocities. In order to resolve the ionization zone around the plate we use a non-uniformly spaced grid in cartesian space obtained from a uniform spacing in the magnetic coordinate $\phi$.

With $z=L_{z}[\exp (\alpha \phi)-1] /[\exp (\alpha)-1]$ and $\alpha=-3$ the grid resolution at the plate is $<0.05 \mathrm{~m}$.

The side walls at $s=0$ and $s=1$ have zero-flux conditions for the plasma and zero-gradient conditions for the neutrals except for the neutral perpendicular velocity $v_{0,1}$ which is fixed to $v_{0,1}=0$. Likewise, $\theta=0$ and $\theta=1$ have zero-flux and zero-gradient conditions for the plasma and the neutrals respectively, except for $v_{0,2}=0$. 


\section{Results and discussion}

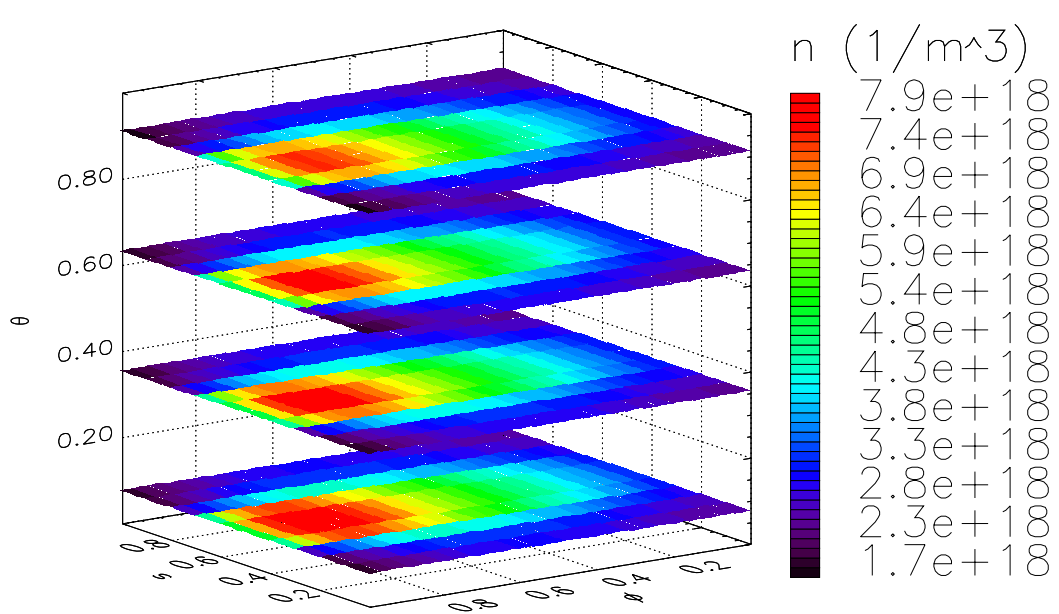

Fig. 1 Plasma density $\left[\mathrm{m}^{-3}\right]$ profiles for a 3D slab in magnetic coordinates with full Navier-Stokes neutrals. Note the density maximum indicating the ionization zone being localized near the target plate at $\phi=1.0$.

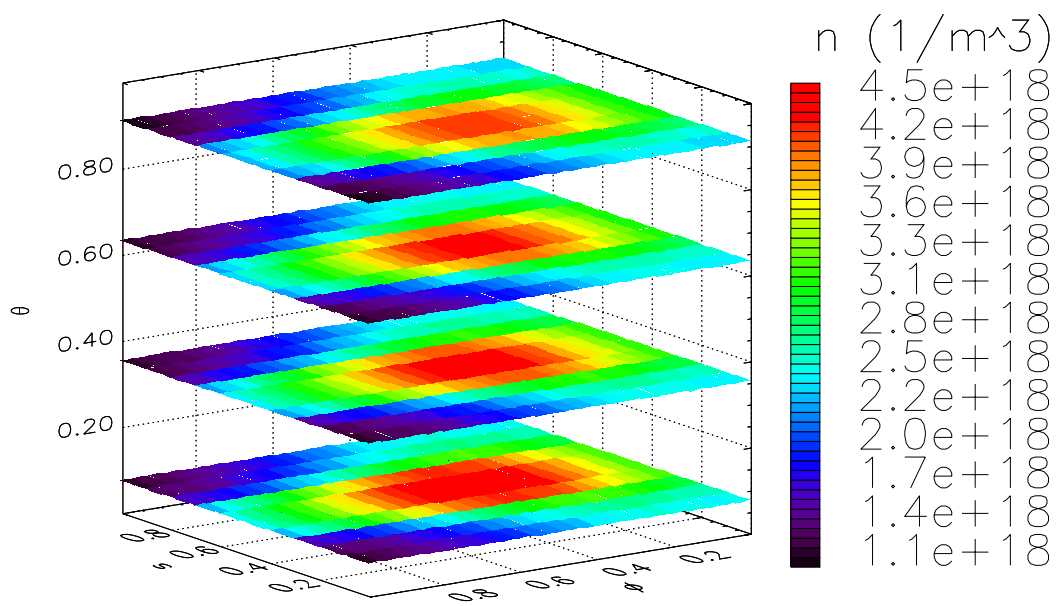

Fig. 2 Plasma density $\left[\mathrm{m}^{-3}\right]$ profiles for a 3D slab in magnetic coordinates with diffusive neutrals.

Neutrals penetrate deeper into the plasma before getting ionized contributing to a less pronounced density maximum.

The three different neutral models were tested and the results are compared to each other. The main characteristics for the plasma properties are found to be similar for all models with very little differences between the parallel and full Navier-Stokes model. For the latter cases, fig.1 illustrates the formation of an ionization zone with enhanced density right in front of the target plate. For both the parallel and full Navier-Stokes model, the momentum recycling coefficient of $R_{M}=0.3$ limits the propagation of neutrals into the plasma and subsequently shifts the ionization zone towards the target plate. The neutral parallel velocities obtained with the diffusive model are higher and thus allow the neutrals to penetrate deeper into the plasma before getting ionized. The result is a more distributed and less pronounced density maximum farther away from the plate as shown in fig. 2 .

High densities correspond to high viscosities for the neutrals. In the diffusive model the neutral momentum is not affected by friction and there are no boundary conditions active. The resulting neutral velocity field reflects the presence of parallel and perpendicular pressure gradients (left part of fig.3). For the parallel Navier-Stokes model, neutral friction leads to an equilibration of their parallel velocities but still allows for variations of the perpendicular components (middle part of fig.3). Subsequently the full Navier-Stokes model (right part of fig.3) 
shows an equilibration of both the parallel and perpendicular velocities due to the high viscosities in front of the target plate, which results in a moderate variation of neutral velocities in the parallel direction on the scale of the neutral mean free path $(\approx 0.15 \mathrm{~m})$.
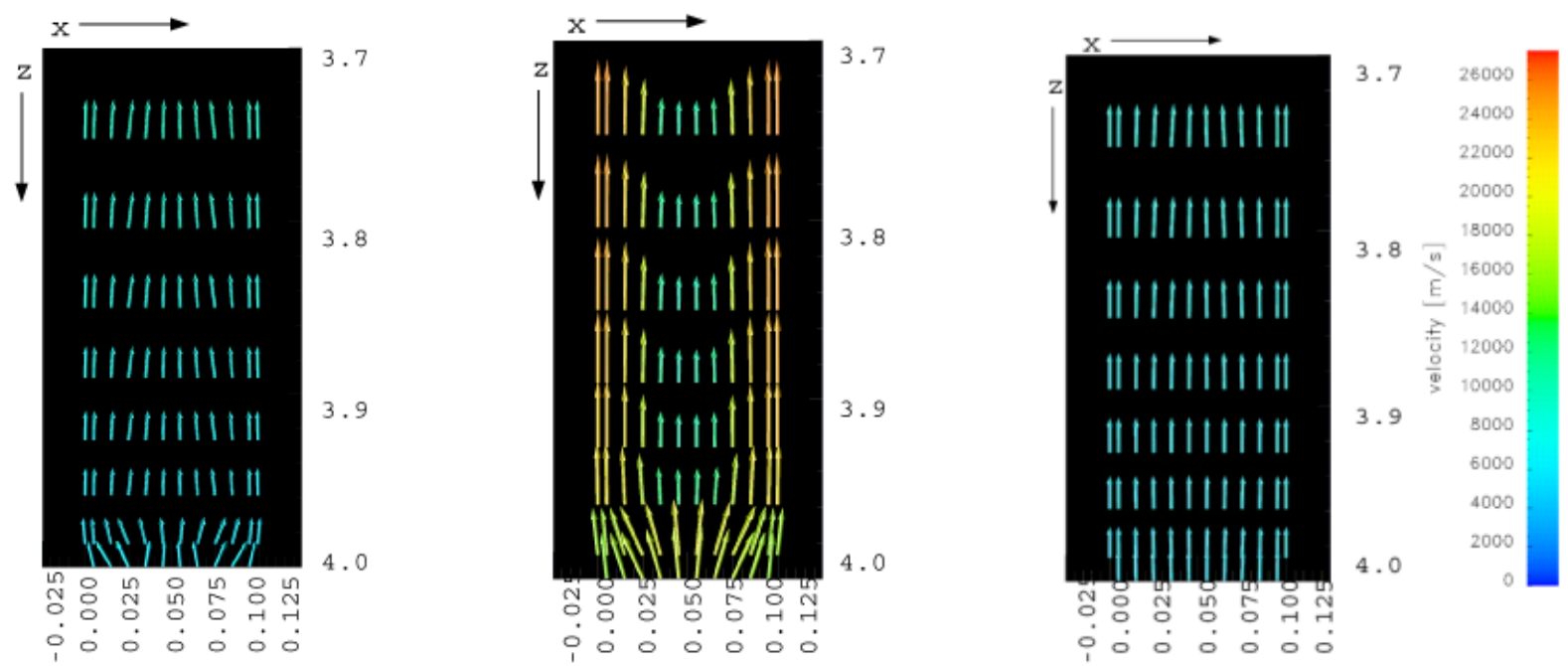

Fig. 3 Neutral velocities $[\mathrm{m} / \mathrm{s}]$ at the target plate $(z=4 \mathrm{~m})$ of a 3D slab as obtained with different neutral models. (left - diffusive, middle - parallel Navier-Stokes, right - full Navier-Stokes)

In BoRiS the system of coupled equations is solved simultaneously with the Newton method utilizing a variety of sophisticated solvers [4]. With respect to more complex and relevant problems like the W7-X stellarator, the above setup also served as a test case for the new parallel version of BoRiS. All results were obtained with an iterative sparse matrix solver GMRES in combination with ILU(0) preconditioning. The runs were performed with a false time-stepping procedure and convergence was found within $\approx 50$ iterations. A robust convergence behaviour can be found with degrees of freedom for varying time stepping procedure and preconditioning.

\section{References}

[1] J. Riemann et al., Journ. of Nuclear Mat. 313-316 1030-1035 (2003).

[2] D. Knoll et al., Phys. Plasmas 3 (1) 293-303 (1996).

[3] A. Grossman et al., Journ. of Nuclear Mat. 220-222 274-278 (1995).

[4] M. Borchardt et al., in Parallel Computational Fluid Dynamics edited by K. Matsuno, A. Ecer, J. Periaux, N. Satofuka and P. Fox Elsevier Science, 459-465 (2003).

This work was performed under the auspices of the U.S. Department of Energy by the University of California, Lawrence Livermore National Laboratory under Contract No. W-7405-Eng-48. 